\title{
Dielectric Characteristic of a Glycerol and a Globular Protein in Solution
}

\author{
Meindinyo R.O.K, Laogun A.A \\ Department of Physics, Niger Delta University Wilberforce Island, Amassoma, Bayelsa State, Nigeria.
}

\begin{abstract}
The dielectric dispersion of a glycerol (lipid) and a globular protein casein (the most abundant protein in milk) have been investigated by means of a resonance technique over the frequency range $0.1 \pm 50$ $\mathrm{MHz}$ for three concentrations and at a mean room temperature of $24 \quad 0.5^{\circ} \mathrm{C}$.The study revealed that casein exhibited beta-dispersion and has while the glycerol did not. The dielectric parameters of the protein like the dielectric increment, relaxation frequency and relaxation time show some dependence on concentration, and the sample studied displayed more than a single relaxation time indicating that the Cole-Cole structural model is better than the single Debye model in explaining its structural properties, whereas this is not the case with the glycerol. The large values of the Cole-Cole spread parameter, $\alpha$ also suggest structural heterogeneity of the casein molecule in solution. The average dipole moment of $386 \pm 16 D$ when compared with other globular proteins could mean some degree of asymmetry of the electric change distribution, of the casein molecules in solution. As for the glycerol it does not exhibit any degree of asymmetry as the curve is almost parallel to the frequency axis.
\end{abstract}

Keywords: Biomolecules, Protein, Lipid, Dielectrics.

\section{Introduction}

Various types of proteins and other bio molecules are known to exist in nature all having different functional roles in living systems. Some protein/bio-moleculesserve as organic catalysts (enzymes), some as transporters of essential materials while others constitute the structure of cells and tissues and so forth. In view of these important roles played by proteins and other bio-molecules in living organisms, interest in the study of the structures and functions of these macromolecules have been in existence for many years in the fields of biophysics and biochemistry. Agricultural engineering/science and related fields. Burubai et $\mathrm{Al}(2012)$. Moreover studies of dielectric properties of a number of proteins were reported to have been carried out by some researches early at the turn of the century, but, no reliable data was obtained until 1931 when some reliable measurements were made by Wyman. This however followed the development of new measurement techniques just about the time.

Various methods now exist for the study of macromolecules and other biological tissues. Some of these are, light scattering, electron microscopy, gel filtration at low ionic strength, calorimetry and nuclear magnetic resonance. In addition, the dielectric method is another well established technique that is in use. Its background was described first by Oncley (1943) and later by Grant (1972) and Grant et al (2005) water of a number of soluble proteins and their dipole moments have been studied by various works (Oncley 1943, Grant, Sheppard and South 1984, Exess et al 1979, Schwan 1957).

Quite a number of globular proteins have been studied by number of workers based on their dielectric dispersions within the radio frequency range. Other studies within their frequency include:carboxyhaemoglobin of horse and pig (Oncley and Shack 1943 and Arrhenius 1943 respectively), Myoglobin (Marcy and Wyman 1938), Serum Albumin (Errera 1932, Oncley 1940), beta - lactoglobulin (Ferry et al 1983), insulin (Oncley 1941, Laogun 1984) Lysozyme (Laogun 1983), Immunoglobulin G (IgG) Laogun (2005).

The work of Oncley and Shack and that of Arrhenius showed that carboxyhaemoglobin of both horse and pig has fairly large dielectric effect. In the case of horse carboxyhaemoglobin, the observed critical frequency was reported to correspond to a relaxation time somewhat too large to be attributed to a spherical molecule with a molecular volume of 50,000 cc but can be explained by assuming an elongated elliposoid of axial ratio of about 1.6 and molecular volume of about $60,000 \mathrm{cc}$ (corresponding protein). The dispersion cure obtained for pig $25{ }^{\circ} \mathrm{C}$ was found to be 1.51 and at $1^{\circ} \mathrm{C}$ it was found to be 1.84 . The dipole moment was also very large (about 730 at $25{ }^{\circ} \mathrm{C}$ and 770 at $0^{\circ} \mathrm{C}$ although the total dipole moment was lower than that of pseudoglobulin and edestin. Its isoionic point of $5.18+0.01$ in dilute $\mathrm{NaCL}$ or KCL was found to be in agreement with the electophoretic isoelectric point of 5.19. it was also reported that the interpretation based on the two relaxation times of an elongated elliposoidal molecule seems somewhat more probable. Results of Shack indicated also a system characteristic to the precision of the measurements by a single relaxation region.

In recognition of the important roles played by proteins in all biochemical or molecular biological reactions, interest in the study of the electrical properties of these macromolecules and other biomolecules and 
cells has for long existed. This is from the understanding that such biochemical reactions are electrical in nature and as such must conform with the laws of physics and electrostatics. That the wide variety of ways in which biomolecules can intereaxt with one another is a result of their electrical properties and therefore an understanding of these properties can yield information about their structures and functions. If the biochemical reactions and molecular behaviours are understood properly, scientist in these fields will be better able (among other things) to design and synthesize possible drugs (the molecular spanners) which could modify selectively the kinetic perturbations which are manifested universally in disease and ageing. Some of such drugs likened as "anti-enzyme guided missiles" might selectively inhibit unwanted or over-reactive enzymes or other proteins while synthetic models might replenish or other proteins while synthetic models might replenish or replace those which are deficient or missing (Elmore 1968).

Studies carried out by various researchers on proteins like insult(a hormone), immunoglobulin $\mathrm{G}$ and such other proteins and the useful results obtained from these studies lend weight to this claim. For instance it is as a result of dielectric studies carried by researchers like Edelman et al (1960) and Laogun (1985) on the immune defense protein immune defense protein immunoglobulin $G$ that showed that the protein is polar in nature and that this polar nature correlates with the bifunctional role of the protein. That one of the fragments (the FC fragment) which is negatively charged can be made to from crystals under certain conditions while the other (Fab fragment) is a positively charge region, a property which assists in its antigen-antibody interactions with predominantly negatively charged proteins and cells. For instance, Therefore knowledge on the distribution of electric charge on biomolecules is important in explaining some of its biological properties.

The needed information about the structure and charge distribution on protein molecules can be obtained by measuring their electrical permittivity or dielectric constant $\left(\varepsilon^{1}\right)$ and conductivity ( $\square$ )in aqueous solution, Oncley (1943), and Laogun et al (1984). From the characteristic dispersion or relaxation of the molecules in solution, the relaxation time and the dielectric increment of the protein can be calculated. The relaxation time is related to the shape and size of the molecule while the dielectric increment is related to the dipole moment.

It is an established fact (Gabriel et al 1983) that interaction of radio-waves with biological media produce heat. The heat dissipated in this process is proportional to $0 \mathrm{E}^{2}$ where 0 is the conductivity and $\mathrm{E}^{1}$ is the electric field. For heterogeneous materials the value of o will very throughout the medium. Also the relative permittivity $\mathrm{E}^{1}$, of the material depends on the nature of the materials under consideration and this together with the conductivity also depends on the frequency and temperature.

In the light of foregoing therefore it was decided to investigate the characteristic dielectric dispersions of certain crystalline samples of the globular protein-casein in solution. This is in order to establish the distinctions between the dielectric dispersions of crystalline samples of this protein and other globular proteins which will aid in explaining its different structural and functional properties. And also, to compare the results with those of other proteins a less complex molecules link propylene glycol.

\section{Dielectric Theory}

Dielectric theory emerged from experimental observation by Michael Faraday in the mid nineteenth centuries and was developed later by Clarke Maxwell. It all resulted from the need for practical insulators since Faraday's early experiments on electrostatics were founded on the isolation of electrostatic charge by dielectric materials which do not conduct the charge away.

The result fundamental in Faraday's experiments was that if in a charged condenser, the air space between the plates is filled with a solid or liquid insulator the potential between the plates is lowered. On the other hand if the potential difference between the plates is kept constant the charge on each plate is increased and hence, the capacity of the condenser $\mathrm{C}$ is increased by a factor independent of the shape or dimensions of the conductor and is solely characteristics of the medium. The factor is called dielectric constant or specific inductive capacity. Hence the fundamental relations for the capacity of a condenser is given by

${ }^{\mathrm{C}}$ dielectric $=\epsilon_{r} C_{\text {vacum }}$

When $\epsilon_{r}$ is called permittivity of the material relative to free space.

i.e. $\epsilon_{r}=\frac{\epsilon}{\epsilon_{0}}$ or $\in=\epsilon_{r} \epsilon_{o}$

Where $\epsilon$ is the permittivity of the medium and $\epsilon_{0}$ is the permittivity of free space.

Where $\in{ }^{\mathrm{dp}}$ is the loss due to dipole polarization. The dielectric constant generally decreases here because the permanent dipoles of the bimolecular cannot match field reversals. Also the proportion of the frictional retarding forces are larger that the orienting ones. In 1929 Debye derived an equation describing the form of this dispersion curve.

This behaviour may be stated formerly by an equation

$\epsilon_{\mathrm{w}}=\epsilon_{\infty}+\int^{\infty} \times(t) \exp .(j w t) d t$ 
In which $\epsilon_{\infty}$ is the value of permittivity of infinite frequency which is a constant and $X(t)$ is some sort of decay factor according to the lagging of polarization behind the applied field. Clearly $\mathrm{x}(\mathrm{t})$ will be connected with the total permittivity such that it can be put in but if it has two chains and above it is termed oligomeric. The component chains are called subunits or protomer

\section{Construction Of Sample Cell}

\section{Materials And Methods}

By a well-established fact that the measuring cell which contains the sample critically affects the accuracy of the measured dielectric parameters. A possible source of error is inductance of the lads of the electrodes, which can be minimized by using short leads. Hence, to obtain very accurate values of the dielectric the method of Laogun et al (1983) was employed to construct the dielectric cell used.

The dielectric cell used consists of a Pyrex glass tube of internal diameter $0.6 \mathrm{~cm}$ terminated at the two ends of the centrally placed upper electrodes of diameter $0.4 \mathrm{~cm}$ mounted on the inner sides of Perspex plates at the ends of the Pyrex glass tube. The Centre of each electrode was soldered at the outside to a short connection lead of about $3 \mathrm{~cm}$ in length. The permittivity of air was measured at different electrode separations, $\mathrm{d}$ of $2 \mathrm{~cm}$, $4 \mathrm{~cm}, 6 \mathrm{~cm}, 8 \mathrm{~cm}, 10 \mathrm{~cm}$ and $12 \mathrm{~cm}$ and a graph of interelectrode capacitance, $\mathrm{c}$ against $\mathrm{d}$ grave a minimum for $\mathrm{C}$ at $6 \mathrm{~cm}$. therefore a cell with inteelectrode separation of $6 \mathrm{~cm}$ was used. The sample solutions were prepared by dissolving an appropriate amount of the powder in distilled water to produce $2.5 \%, 5 \%$ and $10 \%$ solution concentration for the protein, which were considered sufficient to give reliable dielectric data (Laogun 1984).

\section{Determination of $\in$ AND $\sigma$}

The measurement of the real part of the permittivity $\in$ is generally done by measuring the change in capacitance of a condenser, brought about by the introduction of the dielectric between the electrodes. The introduction of the dielectric between the electrodes. The imaginary part $\epsilon$ " is found from the measurement of $\tan \mathrm{s}$, the loss factor arising from the introduction of the dielectric.

Values of the real part of the relative permittivity $\in$ and frequency dependent conductivity $\sigma$, of the protein studied were obtained at frequencies in the range of $0.1-50 \mathrm{MH}_{\mathrm{Z}}$ using a resonance technique (Laogun et at (1986). This technique makes use of a Macroni Q-meter TF1245 working in conjunction with an oscillator TF1246.

The capacitance $\mathrm{C}_{1}$ and the $\mathrm{Q}$-factors $\mathrm{Q}_{1}$ of the test circuit without the specimen were determined at resonance using suitable inductor. And $\mathrm{C}_{2}$ and $\mathrm{Q}_{2}$, the respective values with the specimen connected were again determined at resonance. The effective capacitance, $\mathrm{C}$ of the specimen under study was given by,

The quantities $\epsilon^{1}$, (the relativity permittivity) $\epsilon^{11}$, (the dielectric loss) and $\square$ (the total conductivity) are dependent only on the structural properties as well as on the composition of the specimen and not on the dimensions of the cell.

To determine the cell constant $\mathrm{K}$, and the residual capacitance co for the dielectric cell used in this work, the method of Laogun et al (1983) was employed.

Measurement of the effective capacitance $\mathrm{C}$ were made for distilled water and for air, at frequencies within the range of 0.1 to $50 \mathrm{MHz}$ and were checked by using glycerol and propylene glycerol.

The cell constant $\mathrm{K}$ was calculated from the relation

$$
\mathrm{K}=\frac{\mathrm{C}_{1}-\mathrm{C}_{\mathrm{w}}}{\mathrm{E}^{1} \mathrm{w}}
$$

And the residual capacitance, $\mathrm{Co} \mathrm{Cw}-\mathrm{Kew}$ where $\mathrm{C}_{1}$ is the capacitance obtained and $\mathrm{Cw}$ is the capacitance when water is introduced in the cell. The cell constant obtained in this way was 0.0052620 and the residual capacitance was 0.83470 . The samples were introduced into the cell using a fine hypodermic needle making sure that no air bubbles are trapped.

\section{Sample Preparation}

The experiments were performed using boring casein, (casein isolated from bovine milk), three times crystallized, dialyzed and lyophilized power with $98 \%$ useful and unambiguous information can be obtained at a molecular level as has been previously observed (Grant et al 1978).

The complexiation of structure of the casein and its composition could be used in explaining the origin of the observed dispersion. The marked difference obtained in the change of relative permittivity $\epsilon^{1}$, conductivity $\square$ with frequency may be associated partly with structural differences in terms of amino acid composition, and conformations of the molecule. The high value of the relative permittivity $\epsilon^{1}$ is usually found at the lower frequency part of the frequency range. This maybe explained in general by polarization of the 
molecules at these frequencies (Laogun et al 1983) and the rotation of polar molecules (Grant et al 1978). Also it is believed that in the relaxation region, molecules are on logner able to rotate with the applied electric field.

\section{Results And Discussion}

The result of the relative permittivity of glycerol is given in table 1 where it may be seen that the measured relative permittivity is independent of frequency over the range considered. The data which has been plotted. Table 1,2,3 and 4 show the variation of the dispersion parameters, relative permittivity $\varepsilon^{1}$, conductivity $\alpha$, dielectric loss $\varepsilon^{11}$ and the phase defect, $\tan \alpha$ with frequency respectively for the three concentrations of the globular protein - casein in solution. It obvious that all cases exhibit pronounced frequency dependence of the relative permittivity especially between $1.0 \mathrm{MHz}$ and $20 \mathrm{MHz}$. The Cole-Cole plots of $\epsilon^{1}$ are shown in figure 2,3 and 4 for the three different concentrations of casein solution. Form these plots the Cole-Cole spread parameter, $\alpha$, was obtained. In general the Cole-Cole spread parameter, $\alpha$ varies between 0 and 1 . For a single Debye relaxation, $\alpha=0$. The value of $\alpha$ for all three samples considered varies between 0.13 and o.22 with a mean of $0.17 \pm 0.02$. This indicates that in general, the date exhibit a distribution of relaxation frequencies since $\alpha$ is significantly greater than zero in all cases.

Table 1: variation of dielectric constant $E^{1}$ for glycerol with frequency

\begin{tabular}{|l|l|}
\hline Frequency (MHz) & $\mathbf{E}^{\mathbf{1}} \pm \mathbf{S D}$ \\
\hline 0.1 & $36.1 \pm 0.46$ \\
0.2 & $35.2 \pm 0.39$ \\
0.8 & $36.1 \pm 0.46$ \\
0.9 & $35.2 \pm 0.39$ \\
1.0 & $36.1 \pm 0.46$ \\
3.0 & $36.1 \pm 0.46$ \\
5.0 & $35.2 \pm 0.39$ \\
10.0 & $35.2 \pm 0.39$ \\
20.0 & $35.2 \pm 0.39$ \\
30.0 & $35.2 \pm 0.39$ \\
50.0 & $36.1 \pm 0.46$ \\
\hline
\end{tabular}

$\varepsilon_{\infty}=$ High frequency permittivity;

$\mathrm{SD}=$ Standard Deviation

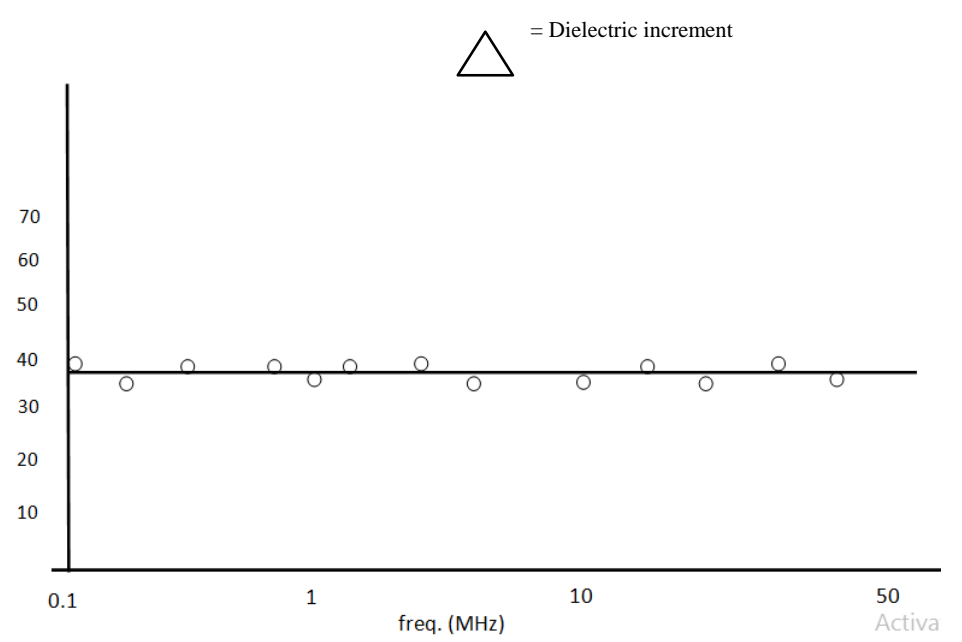

Fig 1: Dispersion of glycerol between $0.1 \& 50 \mathrm{MHz}$

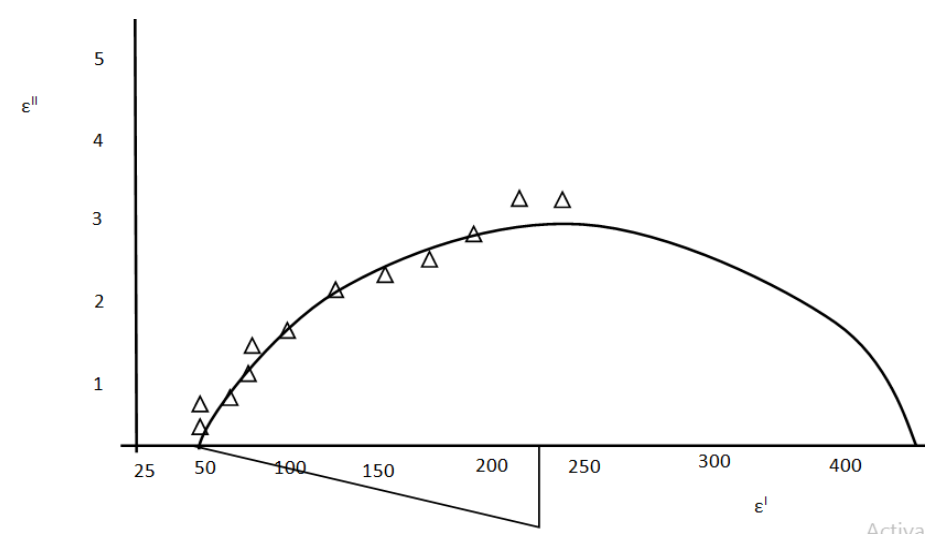

Fig 2: Cole Cole plot of $\varepsilon^{\mathrm{II}}$ against $\varepsilon^{\mathrm{I}}$ for $5 \%$ solution conc. of casein 


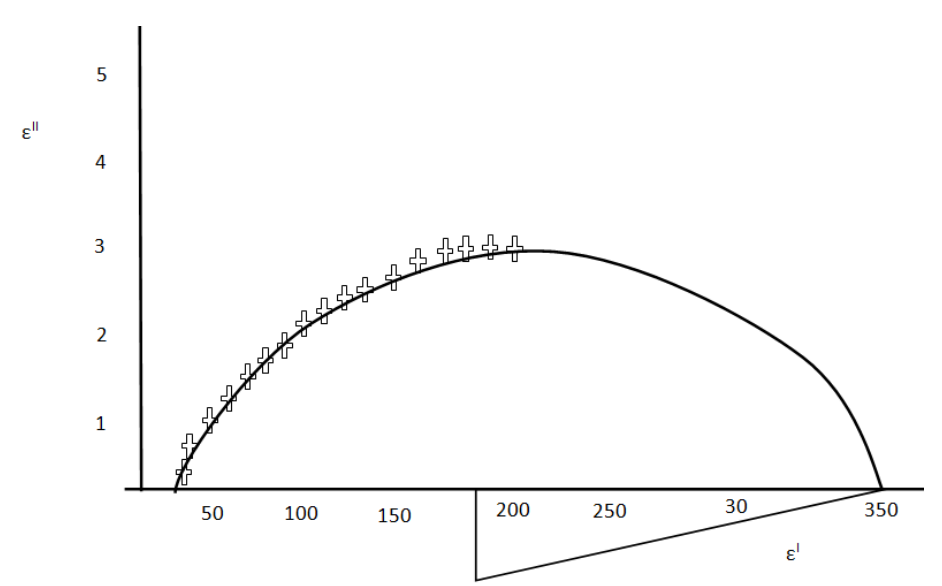

Fig 3: Cole Cole plot of $\varepsilon^{\mathrm{II}}$ against $\varepsilon^{\mathrm{I}}$ for $25 \%$ solution conc. of casein

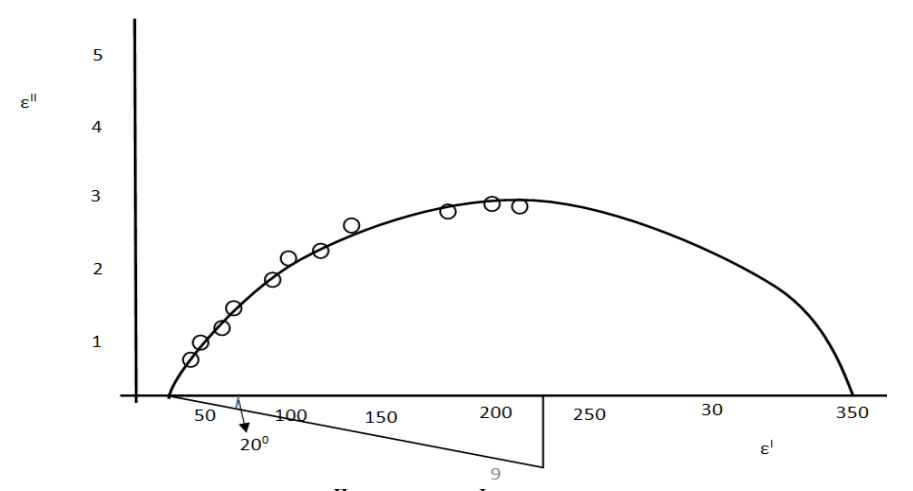

Fig 4: Cole Cole plot of $\varepsilon^{\mathrm{II}}$ against $\varepsilon^{1}$ for $10 \%$ solution conc. of casein

The distribution of relaxation frequencies suggests motions of the dipoles which result in frequency variation of the dielectric constant and the appearance of "dielectric loss" over a broad band of frequencies. When the field is changing sufficiently fast, the molecular forces impeding the dipoles orientation dominate and the dipoles become unable to follow the changes. At these frequencies the orientation of the permanent dipoles no longer contributes to the dielectric constant. Moreover, in certain frequency bands a phase lag between the field and dipole orientation develops and energy is drawn from the electrical source by the material and is dissipated as heat. This phenomenon is described by a complex representation of the dielectric permittivity $\varepsilon^{*}$ given by

$\mathrm{E}^{*}=\varepsilon^{*}-\mathrm{j} \varepsilon^{*}$

Where $\varepsilon^{1}$ is the real of the dielectric constant and $\varepsilon^{\mathrm{ii}}$ is the imaginary part and is known as the dielectric loss which is a parameter describing the motion of the electric change, that is, a conduction phenomenon. Dielectrics are often known to display conduction on the displacement current, but also from actual charge transport (e.g. ionic conduction as in electrolytes). The conductivity is usually represented bycand its effect would be to an additional term to the dielectric loss from these plots. It can be seen that from the values of $\square$ show on table 8 that casein molecules in solution do not exhibit a single Debye behaviour since $\square$ is significantly different from zero for all three samples. In the case of glycerol molecule there is no structural heterogeneity as it is a simple molecule. It can therefore be said that the Cole-Cole model gives a better fit for the measured dielectric parameters which shows a spread of the relaxation times and frequencies for casein and there is no structural hetero glycerol for this biomolecules structural glycerol is a simple polyol compound. It is a colorless, viscous liquid that is sweet-tasting and non-toxic. The glycerol backbone is found in all lipids known as triglycerides.

\section{Structure of a Glycerol}

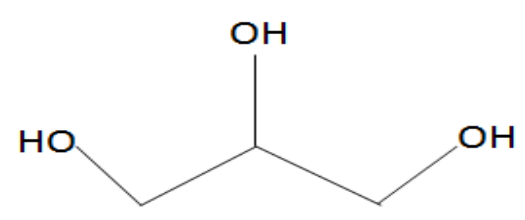


These may be related to structural and electrical properties of the molecules in solution. Actually the need for such accurate data on the dielectric parameters for proteins and other biological materials has been will recognized as this will help in proper understanding of the structure and functions of these biomolecules at the molecular level (Laogun 2005, Mckenzie 2005). During the last five decades, dramatic progress has been made in the knowledge of the biochemistry of proteins especially globular proteins as related to their dielectric properties. A remarkable picture is gradually emerging of their structure and the relationship of these structures to their biological functions. This study

Table 2: Variation Of Phase Defect Tan S, With Frequency For 3 Concentrations Of Caesine Solution.

\begin{tabular}{|c|c|c|c|}
\hline Frequency $\left(\mathrm{MH}_{\mathrm{Z}}\right)$ & \multicolumn{3}{|c|}{ Tan $\mathrm{s}$} \\
\hline & $2.5 \%$ & $5 \%$ & $10 \%$ \\
\hline 0.1 & 1.216 & 1.31 & 1.19 \\
\hline 0.3 & 1.18 & 1.31 & 1.20 \\
\hline 0.5 & 1.34 & 1.39 & 1.52 \\
\hline 0.8 & 1.997 & 1.85 & 1.42 \\
\hline 0.9 & 1.43 & 1.48 & 2.18 \\
\hline 1.0 & 1.72 & 1.74 & 2.14 \\
\hline 1.5 & 1.49 & 1.72 & 1.36 \\
\hline 2.0 & 1.15 & 1.70 & 1.12 \\
\hline 3.0 & 1.12 & 1.32 & 1.27 \\
\hline 4.0 & 1.03 & 1.23 & 1.36 \\
\hline 5.0 & 0.84 & 0.86 & 1.20 \\
\hline 7.0 & 0.89 & 0.77 & 0.96 \\
\hline 8.0 & 0.71 & 0.84 & 0.92 \\
\hline 10.0 & 0.68 & 0.89 & 0.86 \\
\hline 15.0 & 0.54 & 0.40 & 0.82 \\
\hline 20.0 & 0.44 & 0.65 & 0.81 \\
\hline 30.0 & 0.53 & 0.6 & 0.71 \\
\hline 50.0 & 0.39 & 0.67 & 0.74 \\
\hline
\end{tabular}

TABLE 3: Variation Of Relative Permittivity $E^{i}$ With Frequency For Three Concentrations Of CaseinSolutions.

\begin{tabular}{|l|l|l|l|}
\hline Frequency $\left(\mathrm{MH}_{\mathrm{Z}}\right)$ & Relative permittivity, $\mathrm{E}^{1}$ & \%conc. $=5.0$ & \% conc. $=2.5$ \\
\hline & 125.6 & 149.9 & 193.4 \\
\hline 0.1 & 125.2 & 149.3 & 193.4 \\
\hline 0.3 & 124.6 & 148.2 & 192.9 \\
\hline 0.5 & 124.2 & 147.9 & 192.9 \\
\hline 0.8 & 124.2 & 147.9 & 183.4 \\
\hline 0.9 & 122.0 & 145.4 & 184.9 \\
\hline 1.0 & 120.0 & 142.4 & 154 \\
\hline 1.5 & 116.2 & 129.9 & 145.4 \\
\hline 2.0 & 114.0 & 130.9 & 135.9 \\
\hline 3.0 & 112.0 & 120.9 & 126.4 \\
\hline 4.0 & 110.2 & 106.9 & 116.9 \\
\hline 5.0 & 104.4 & 107.4 & 107.4 \\
\hline 7.0 & 98.9 & 99.9 & 99.9 \\
\hline 8.0 & 97.9 & 98.9 & 88.9 \\
\hline 10.0 & 88.9 & 98.9 & 98.9 \\
\hline 15.0 & 78.9 & 88.9 & 98.9 \\
\hline 20.0 & 78.9 & 78.9 & 78.9 \\
\hline 30.0 & 88.9 & 98.9 & 98.9 \\
\hline 50.0 & & \\
\hline
\end{tabular}

TABLE 4: Variation Of Relative Permittivity $C^{\mathrm{i}}$ With Frequency For Three Concentrations Of Casein Solutions

\begin{tabular}{|l|l|l|l|}
\hline Frequency $\left(\mathrm{MH}_{\mathrm{Z}}\right)$ & \multicolumn{3}{|c|}{ Relative Permittivity $\mathrm{E}^{\mathrm{I}}$} \\
\hline & \%Conc. $=2.5$ & \%Conc. $=5.0$ & \% Conc. 10.0 \\
\hline 0.1 & 125.6 & 149.9 & 193.4 \\
\hline 0.3 & 125.2 & 149.3 & 193.4 \\
\hline 0.5 & 124.6 & 148.2 & 192.9 \\
\hline 0.8 & 124.2 & 147.9 & 192.9 \\
\hline 0.9 & 124.2 & 147.9 & 183.4 \\
\hline 1.0 & 122.0 & 145.4 & 192.9 \\
\hline 1.5 & 120.0 & 142.4 & 192.9 \\
\hline 2.0 & 116.2 & 129.9 & 183.4 \\
\hline 3.0 & 114.0 & 130.9 & 184.9 \\
\hline 4.0 & 112.0 & 120.9 & 154 \\
\hline 5.0 & 110.2 & 106.9 & 145.4 \\
\hline 7.0 & 104.4 & 107.4 & 99.9 \\
\hline 8.0 & 98.9 & 107.4 & 88.9 \\
\hline
\end{tabular}


Dielectric Characteristic Of A Glycerol And A Globular Protein In Solution

\begin{tabular}{|l|l|l|l|}
\hline 10.0 & 97.9 & 99.9 & 98.9 \\
\hline 15.0 & 78.9 & 98.9 & 88.9 \\
\hline 20.0 & 88.9 & 88.9 & 98.9 \\
\hline 30.0 & 78.9 & 78.9 & 78.9 \\
\hline 50.0 & 88.9 & 98.9 & 98.9 \\
\hline
\end{tabular}

TABLE 5: Variation of Dielectric Loss 6 " With Frequency For Three Concentrations Of Casein Solution

\begin{tabular}{|l|l|l|l|}
\hline Frequency $\left(\mathrm{MH}_{\mathrm{Z}}\right)$ & \multicolumn{1}{|c|}{ Dielectric loss E' } & $10 \%$ \\
\hline & $2.5 \%$ & $5 \%$ & 231.3 \\
\hline 0.1 & 152.12 & 200.5 & 222.4 \\
\hline 0.3 & 149.63 & 204.5 & 241.6 \\
\hline 0.5 & 233.16 & 194.8 & 252.8 \\
\hline 0.8 & 242.02 & 246.81 & 250.2 \\
\hline 0.9 & 234.69 & 241.21 & 249.8 \\
\hline 1.0 & 233.44 & 238.51 & 247.7 \\
\hline 1.5 & 240.10 & 234.5 & 241.7 \\
\hline 2.0 & 233.16 & 231.02 & 240.3 \\
\hline 3.0 & 247.0 & 244.28 & 235.5 \\
\hline 4.0 & 145.5 & 226.6 & 295.0 \\
\hline 5.0 & 83.34 & 108.3 & 284.7 \\
\hline 7.0 & 76.38 & 150.9 & 197.5 \\
\hline 8.0 & 72.69 & 127.5 & 192.8 \\
\hline 10.0 & 41.59 & 131.4 & 93.5 \\
\hline 15.0 & 49.55 & 42.2 & 80.12 \\
\hline 20.0 & 49.84 & 84.4 & 87.10 \\
\hline 30.0 & 44.28 & 45.3 & 79.3 \\
\hline 50.0 & 46.77 & 36.8 & \\
\hline
\end{tabular}

\section{Reference}

[1]. Burubai, W., Meindinyo, R.O.K(2013), Effect of moisture and frequency variations on the dielectric properties of Africa nutmeg (monodoramyristica) seeds.

[2]. Burubai, W. Dagogo G.W, Meindinyo R.O.K,(2012) Strain energy variability as a function of moisture content, preheating temperature and loading rate on African nutmeg seed during cracking.

[3]. Henry, F., L. Costa, and F. Lakkis. 2003. Free and/or bound water by dielectric measurements. Food chemistry.

[4]. Afzal, A,S. Mousavi, and M.Khademi. (2010). Estimation of leaf moisture content by measuring content by measuring the capacitance.

[5]. Anderson, J. C. (1967): Dielectrics (London: Chapman and Hill Ltd.): PP 15-19, 49-50.

[6]. Bleany, B. I. and Bleany, B. (1976): Electricity and Magnetism (Oxford: University Press): PP 20-25, 41-43.

[7]. Coelho, R. (1979): Physics of Dielectrics for the Engineers (New York: Elsevier Scientific pub Co.), pp 60-68.

[8]. Cole, K. S. and Cole, R. H. (1914): Dispersion and Absorption in Dielectrics. J. Chem. Phys. Vol. 9, pp. 341 - 351

[9]. Conn, S. (1976): Outline of Biochemistry (USA: John Wiley \& Sons Inc.), pp 73 -79.

[10]. Debye, P. (1929): Polar Molecules ( New York: Chemical Catalog Co.) Ch. 5.

[11]. Elmore, D. T. (1968): Peptides and proteins (Cambridge: University Press): pp144 - 246.

[12]. Epstein, H. T. (1963): Elementary Biophysics (Massachusetts: Adosspm-Wesley): pp 23-26.

[13]. Essex, C. G., South, G. P., Sheppard, R. J. and Grant, E. H. (1975): A Bridge Technique for Measuring the permittivity of a Biological Solution between 1 and $100 \mathrm{MHz}$. J. of phys. E: Scientific Instrumentation Vol. 8, pp. 385 - 389.

[14]. Ferrapo, V. C. A. (1970): Electromagnetic theory (Edinburg: Oliver and Boyd) pp 1520.

[15]. Frohleek, H. (1978): Theory of Dielectrics (Oxford, University Press): pp 62 -78.

[16]. Frohleek, H. (1958): Electrical Interactions in Molecular Biophysics (New York: Academic press): pp 67 -79

[17]. Gabler, R. (1978): Electrical Interactions in Molecular Biophysics (New York: Academic Press): pp 67 - 79

[18]. Grant, E. H. (1972): Solid State Biophysics: McGraw-Hill Advanced Physics Monograph Series ed. S. J. Wyard (New York: McGrae-Hill)

[19]. Grant, E. H.; Sheppard, R. J. and South (1978): Dielectric Behaviour of Molecules in Solution (Oxford: University Press) Ch. 2, 5, 6.

[20]. Hey, D.H (1996) ed.Kingzetts Chemical Encyclopaesia (London: Bailliere\& Cassel) p 178.

[21]. Jennesss, R. (1970) : Milk Proteins: Chemistry and Molecular Biology ed. H.A Mckenzie (New York: Academic), Ch. 2.

[22]. Laogun, A.A; Ajayi, N.O.; Okafor, L.O and Osamo, N.O. (1983): Dielectric Characteristics of parked human erythrocytes with haemoglobins F, AA, AS, SS. Phys. Med. Biol., Vol. 9, No. 4, pp 341-

[23]. Laogun, A.A. (1984): Dielectric properties of eggwhite, iysozymeinsolution. Phy. Med. Biol., Vol. 12, No. 2, pp 241-245. 